\title{
Distribution of Thoracic Aortic Calcifications in Patients Undergoing Coronary Artery Bypass Grafting
}

\author{
Adem Ilkay Diken, MD*, Adnan Yalçınkaya, MD, Sertan Özyalçın, MD \\ Department of Cardiovascular Surgery, Hitit University Faculty of Medicine, Corum, Turkey
}

\begin{abstract}
Background: In procedures involving surgical maneuvers such as cannulation, clamping, or proximal anastomosis where aortic manipulation is inevitable, a preliminary assessment of atherosclerotic plaques bears clinical significance. In the present study, our aim was to evaluate the frequency and distribution of aortic calcifications in patients undergoing coronary artery bypass grafting (CABG) surgery to propose a morphological classification system. Methods: A total of 443 consecutive patients with coronary artery disease were included in this study. Preoperative non-contrast enhanced computed tomography images, in-hospital follow-up data, and patient characteristics were retrospectively evaluated.

Results: Whereas $33 \%$ of patients had no calcifications at any site in the aorta, $7.9 \%, 75.4 \%$, and $16.7 \%$ had calcifications in the ascending aorta, aortic arch, and descending aorta, respectively. Focal small calcifications were the most common type of lesions in the ascending aorta (3.9\%), whereas 9 patients $(1.4 \%)$ had porcelain ascending aorta. We defined four types of patients with increasing severity and extent of calcifications.

Conclusions: Based on the frequency and distribution of calcifications in the thoracic aorta, we propose a classification system from least to most severe for coronary artery disease patients who are candidates for CABG.

Copyright @ 2017 Science International Corp.
\end{abstract}

\section{Key Words}

Calcification • Coronary artery bypass grafting • Aorta

\section{Introduction}

Atherosclerotic plaques of the ascending aorta represent a potential risk factor for certain complications during cardiac surgery [1, 2], particularly for cerebrovascular events (CVEs) due to cerebral embolization of debris that breaks off from the atheromatous plaque [3]. Aortic atheromas are characterized by a minimum intimal thickening of $2 \mathrm{~mm}$ as well as increased echogenicity or density. Increasing plaque thickness with resultant protrusion into the lumen with mobile fragments is associated with a significantly increased risk of stroke. According to the French Aortic Plaque in Stroke Group statement, the presence of a plaque $>4$ $\mathrm{mm}$ in thickness is associated with a significant risk of stroke [4]. Thus, in procedures involving unavoidable aortic manipulation such as cannulation, clamping, or proximal anastomosis, pre-assessment of atherosclerotic aortic plaques should be undertaken [1,5]. Diagnostic modalities commonly used for this purpose include intraoperative epicardial echocardiography, computed tomography (CT), and transesophageal echocardiography (which is routine for all heart cases in most United States hospitals).

Aortic atherosclerosis frequently co-exists with aortic calcifications. Previous findings also suggest an association between cardiovascular adverse events and aortic calcifications, which is a common coincidental finding on chest X-rays or CT imaging studies performed for other reasons [6]. Patients with coro-

\footnotetext{
* Corresponding Author:

Adem Ilkay Diken, MD

Department of Cardiovascular Surgery

Hitit University Faculty of Medicine

Camlik Cad. No: 2 Corum, 19100, Turkey

Tel.: +90 530687 3315; Fax: +90 364219 3001; E-Mail: ademilkay@gmail.com
}

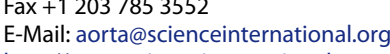

http://aorta.scienceinternational.org

\author{
Published by Science International Corp \\ ISSN 2325-4637 \\ Accessible online at: \\ http://aorta.scienceinternational.org
}


nary artery disease have an increased frequency and extent of such calcifications [7]. Therefore, the objective of the current study was to determine the incidence and distribution of calcifications of the thoracic aorta in patients undergoing elective coronary artery bypass grafting (CABG). Additionally, we propose a new method for the classification of calcifications of the thoracic aorta for this patient population.

\section{Materials and Methods}

A total of 433 CABG surgery patients evaluated by thoracic $\mathrm{CT}$ for any reason (mainly pulmonary) between 2012 and 2016 were included. Patient data were retrospectively evaluated using the hospital database and surgery notes. Patients underwent a CT imaging study of the thorax without intravenous contrast enhancement prior to surgery using the same device and imaging protocols. The distribution and localization of aortic calcifications in acquired images were recorded.

\section{Computed Tomography Protocol}

A 128-slice multidetector spiral thoracic CT scan (Optima, GE Medical Systems, Waukesha, WI) was performed during a 15-s period of apnea at the end of a normal inspiration. Exposures were taken at $120 \mathrm{kV}$ and 200-250 mA. Scanning was performed from the apex of the lung to the diaphragm level.

\section{Statistical Analysis}

Demographic characteristics were expressed as mean \pm standard deviation and analysed using Pearson's Chi-square or Fisher's exact tests. Mann-Whitney $U$ tests were performed for comparisons of continuous variables. Potential independent predictors of outcome were identified by univariate analyses. A $p<0.05$ was considered statistically significant.

\section{Results}

Whereas $33 \%$ of patients had no aortic calcifications, $10.8 \%$ had isolated calcifications in the descending aorta. Isolated calcifications of the ascending aorta were rare (6 patients, 1.4\%). Multifocal calcifications were present in most patients (237 patients, 54.7\%).

The most frequent sites of calcifications were the arch, the descending, and ascending aorta $(75.4 \%$, $16.7 \%$, and $7.9 \%$, respectively). Most calcifications in the aortic arch were found around branching points. The three branching points from the arch (i.e., brachiocephalic artery, left common carotid artery, and left subclavian artery) were categorized as segment 1 , 2 , and 3, respectively (Figure 1). Most calcifications occurred in the third segment of the aortic arch (32.9\%) followed by the second (20.6\%) and first (15.3\%) segments. In addition to calcifications at branching points, there were also smaller foci of calcifications with somewhat variable anatomical locations in a single CT cross-section as well as more extensive patchy areas of calcification. Table 1 shows detailed information on the anatomical distribution of calcifications.

No preoperative characteristics were significantly different between patients with porcelain aortas and those with normal aortas (Table 2).

The most frequent type of calcifications in the ascending aorta were small, focal calcifications (3.4\%) with no specific disposition for an anatomical site, as was the case for patchy calcifications. There were 9 pa-

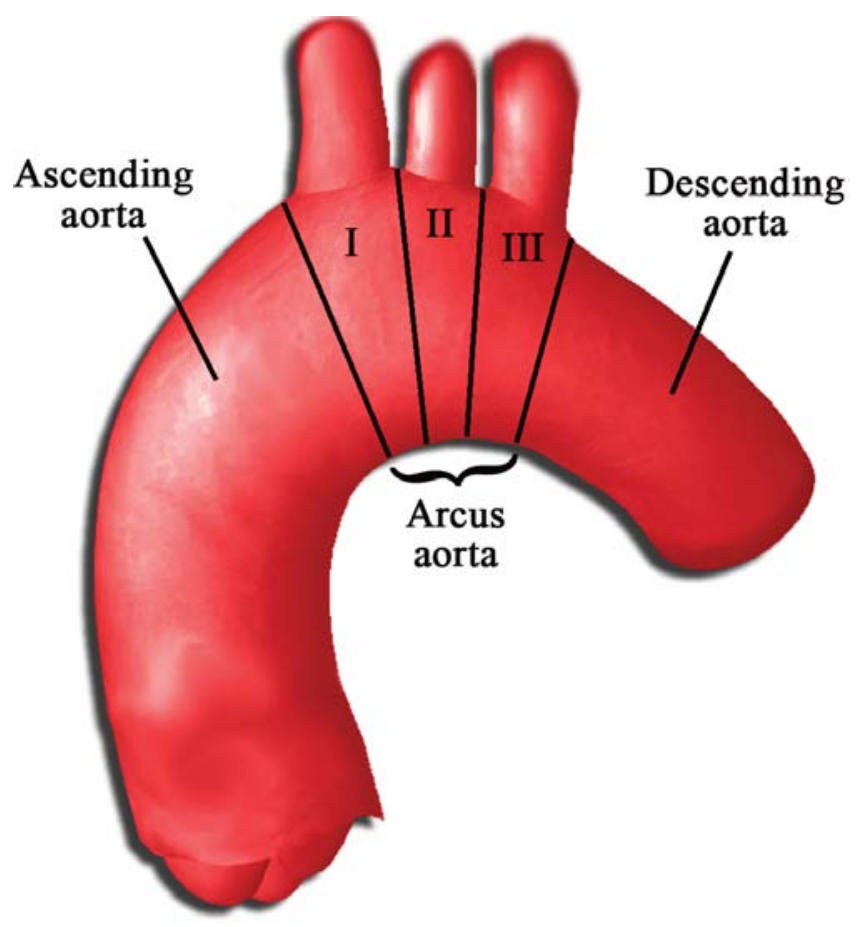

Figure 1. Regions of the aortic arch. 
Table 1. Site of calcifications on different aortic localizations.

Site of calcifications

Number of lesions Percentage in total lesions (\%)

Ascending aorta

Focal calcifications in ascending aorta

$25 \quad 3.93$

Patchy calcifications in ascending aorta

$16 \quad 2.52$

Porcelain ascending aorta

$9 \quad 1.41$

Aortic arch

Region 1 of aortic arch

Ostium of brachiocephalic artery and neighboring aortic arch

Region 2 of aortic arch

Ostium of left common carotid artery and neighbouring aortic arch

Region 3 of aortic arch

Ostium of left subclavian artery and neighbouring aortic arch

Focal calcifications in aortic arch

Patchy calcifications in aortic arch

Porcelain aortic arch

$\begin{array}{ll}12 & 1.88 \\ 19 & 2.99 \\ 11 & 1.73\end{array}$

Descending aorta

Focal calcifications in descending aorta

Patchy calcifications in descending aorta

$22 \quad 3.46$

Porcelain descending aorta

$12 \quad 1.88$

Descending aorta/connection site of ductus arterious

Table 2. Preoperative variables and aortic calcification in univariate analysis.

\begin{tabular}{lccc}
\hline & $\begin{array}{c}\text { Porcelain } \\
\text { aorta }(\mathbf{N}=\mathbf{9})\end{array}$ & $\begin{array}{c}\text { Normal aorta } \\
(\mathbf{N}=143)\end{array}$ & P Value \\
\hline Age (years) & $60.1 \pm 18.2$ & $58.3 \pm 17.1$ & NS \\
Sex & $3(\% 33.33)$ & $47(\% 32.86)$ & NS \\
Female & $6(\% 66.66)$ & $96(\% 67.13)$ & NS \\
Male & $7(\% 77.77)$ & $103(\% 72.02)$ & NS \\
Hypertension & $6(\% 66.66)$ & $78(\% 54.54)$ & NS \\
$\begin{array}{l}\text { Hyperlipidemia } \\
\text { Diabetes mellitus }\end{array}$ & $3(\% 33.33)$ & $40(\% 27.97)$ & NS \\
$\begin{array}{l}\text { Chronic obstructive } \\
\text { pulmonary disease }\end{array}$ & $4(\% 44.44)$ & $58(\% 40.55)$ & NS \\
$\begin{array}{l}\text { Peripheral arterial } \\
\text { disease }\end{array}$ & $1(\% 11.11)$ & $15(\% 10.48)$ & NS \\
$\begin{array}{l}\text { Chronic renal } \\
\text { failure }\end{array}$ & 0 & $1(\% 0.69)$ & NS \\
\hline NS = not significant. & & & \\
\hline
\end{tabular}

tients (1.4\%) with annular calcifications involving more than $75 \%$ of the diameter of the ascending aorta (i.e., porcelain aorta); none of these patients experienced a neurological adverse event.

In two patients $(0.5 \%)$ with no calcifications or thickening on $\mathrm{CT}$ images of the ascending aorta, extensive palpable areas of firmness were detected during the intraoperative examination of the ascending aorta. Based on the potential presence of atherosclerotic plaques in such areas, aortic manipulation was avoided and proximal anastomoses were performed on the left internal mammarian artery or intact right brachiocephalic trunk.

An assessment of CT images allowed the classification of patients with aortic calcifications into several groups (Figure 2) as follows:

Type 1. Single area of calcification at the origin of the brachiocephalic artery, left common carotid artery, or left subclavian artery.

Type 2. Calcification in at least two origins of the brachiocephalic artery, left common carotid artery, 


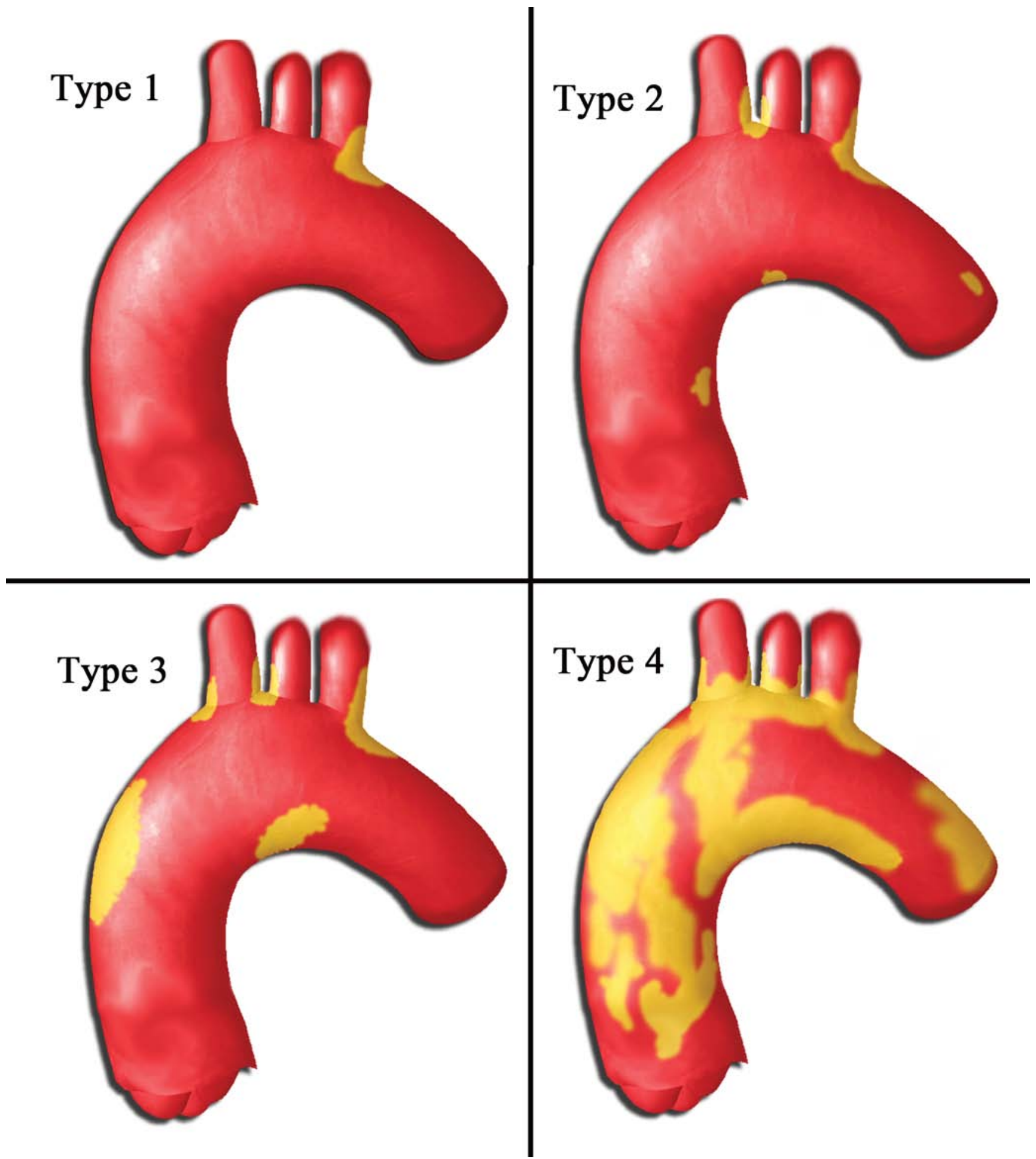

Figure 2. Proposed classification system based on the severity and extent of aortic calcifications. 
Table 3. Patient distribution among types.

Number of patients Percentage (\%)

\begin{tabular}{lcc} 
Normal & 143 & 37.04 \\
Type 1. & 99 & 25.64 \\
Type 2. & 94 & 24.35 \\
Type 3. & 41 & 10.62 \\
Type 4. & 9 & 2.33 \\
Total * & 386 & 100 \\
\hline
\end{tabular}

* Isolated descending aortic calcification was detected in 47 of 433 patients. These patients were not classified into the groups and were not were not accounted for in the total number.

or left subclavian artery and/or small foci of calcifications in the ascending or aortic arch.

Type 3. Presence of non-annular, patchy calcifications in the ascending aorta in addition to calcifications at the origin of the brachiocephalic artery, left common carotid artery, or left subclavian artery.

Type 4. Extensive annular ascending aortic calcifications frequently accompanied by significant calcifications at other areas.

Table 3 shows the distribution of the number of patients in each group. The severity and extent of calcifications increased from the top to the bottom.

The severity and distribution of calcifications in the descending aorta was independent of those of the ascending and aortic arch; even in patients with complete absence of calcifications in other thoracic aortic segments, some had annular calcifications of the descending aorta. Therefore, a separate category of calcification in the descending aorta in addition to the above-mentioned types may provide additional clarity on the site of calcifications. Furthermore, the absence of information on the descending aorta can be considered acceptable in this group of coronary artery disease patients because it does not have direct consequences on the surgical protocol.

\section{Discussion}

The presence of severe calcifications in the ascending aorta and aortic arch may alter the course of a scheduled cardiac surgical procedure and is associated with an increased risk of morbidity and mortality $[2,8]$. A change in the site of cannulation and type of graft upon detection of aortic calcifications after sternotomy may require the use of another myocardial protection technique or intra-aortic balloon occluder [9]. Therefore, these lesions should ideally be identified prior to the surgical procedure to allow readiness for all scenarios. In our unit, all cardiac surgery patients have undergone routine $\mathrm{CT}$ scanning of the thorax for the past 3 years to evaluate the ascending aorta or identify possible pulmonary pathologies before surgery. Among our patients, $67 \%$ had calcifications in the thoracic aorta, whereas only 9 (2.3\%) had porcelain ascending aorta. Thus, we proposed a morphological classification system based on the distribution of calcified lesions.

CVEs are one of the most serious complications of CABG surgery, with a reported incidence between $1.4 \%$ and $3.8 \%$ [10]. In most studies comparing percutaneous coronary intervention ( $\mathrm{PCl}$ ) with $\mathrm{CABG}$, surgical procedures were more frequently associated with CVEs $[11,12]$. Etiological factors for CVEs occurring after CABG include intracranial cerebral atherosclerosis, extracranial carotid artery stenosis, atherosclerosis of the thoracic aorta, and other causes associated with cardiopulmonary bypass itself [13].

A limited number of previous studies have examined aortic calcifications in similar groups of patients. Information on the incidence of porcelain aorta in coronary artery disease patients is also scarce. In a study by Nishi et al. examining aortic calcifications in patients undergoing a variety of cardiac procedures [9], the incidence of severe calcifications (described as porcelain aorta, Type 4 in this study) in the ascending aorta was almost 3 -fold higher $(1.41 \%$ vs. $4.33 \%)$ than that in our study. In this previous study, the presence of severe calcifications in the ascending aorta was associated with preoperative renal dysfunction, dialysis, and aortic valve stenosis, although only aortic stenosis emerged as a predictor of severe calcification of the ascending aorta in multivariate analysis [9]. On the other hand, no preoperative parameters had predictive value for severe calcifications of the ascending aorta in our study. This may be due to the limited number of parameters examined or the smaller number of patients with renal dysfunction.

Intraoperative epiaortic ultrasound examination of the aorta is another valuable tool for detecting aortic 
atherosclerosis [14]. However, preoperative detection of severe porcelain aorta cannot be considered equivalent to its identification only after the sternum is opened, as the latter represents a much less predictable surgical course both for the patient and surgeon. However, preoperative detection of these lesions with detailed examination would allow preparation of the cannulation site (femoral, subclavian artery, or axillary artery) and the planning of logistic arrangements. In 9 patients with porcelain aorta in our study, the site of arterial cannulation was prepared prior to surgery based on the results of preoperative assessments, and CABG on a beating heart was performed without complications or manipulation of the aorta. On the other hand, detection of porcelain aorta in an elderly patient with two/three vessel disease may allow the heart team to opt for $\mathrm{PCl}$ rather than CABG in appropriately selected cases based on a risk-benefit analysis. Therefore, despite its cost, preoperative use of $\mathrm{CT}$, at least in select patient populations, may be a rational approach. In countries with a relatively low cost of CT imaging (i.e., $€ 18$ for a non-contrast enhanced chest CT scan in Turkey), its routine use may also be considered a viable option, as it may provide certain advantages.

Although international classification systems have been developed for certain pathological conditions of the aorta such as aneurysms or dissections, no consensus-based classification system for atherosclerotic aortic disease exists. Classification of calcified lesions of the aorta may allow sharing of information on a common ground before and during surgery. Amorim et al. divided thoracic aortic calcifications into two types as a means for such a classification [15], with Type 1 and Type 2 calcifications involving lesions in the ascending aorta and descending and/or aortic arch, respectively. Type 1 was further divided into the sub-groups of Type $1 \mathrm{a}$, in which clamping is unachievable, and Type $1 \mathrm{~b}$, in which adequate tissue exists for clamping. Accordingly, porcelain aorta was considered Type 1a, whereas patchy calcifications with areas of no calcification allowing clamping was considered Type $1 \mathrm{~b}$. Although this is a practical approach, it is essentially a functional classification, and the authors emphasized that it was not an all-encompassing classification system for all localizations.
On the other hand, the classification proposed here was developed based on the frequency of occurrence and severity of calcifications, which increases as one advances from Type 1 to Type 4. Type 1, the most frequent pattern, includes patients who have no lesions in the ascending aorta or body of the aortic arch but have small foci of calcifications in the branching points of the aortic arch. Type 2 consists of small foci of calcifications in the aortic arch or ascending aorta. Type 3 consists of patchy areas of calcifications along with arch calcifications. Finally, Type 4 refers to severe circumferential calcifications. Thus, this classification system considers not only the site but also the size and severity of the lesions. This may be used as a guide for further studies aiming to support a common terminology.

A major limitation of our study is the inclusion of elective CABG patients only. Also, soft atheromatous plaques or penetrating ulcers of the aorta could not be evaluated, as non-contrast enhanced CT was utilized. It should be noted that although calcification constitutes a major component of atherosclerosis of the aorta, non-calcified soft plaques may also cause stroke. During retrospective analysis, we observed that intraoperative epiaortic ultrasound was irregularly used, as surgeons preferred its use only in suspected ascending aortas instead of routine practice. Therefore, we did not use this data to prevent potential bias. Despite these limitations, our findings allowed the development of a useful and practical classification of thoracic aortic calcifications.

In conclusion, this study provided information on the frequency and distribution of calcifications in the thoracic aorta in patients undergoing elective $C A B G$. Using this information, we proposed a classification system grouping patients from mild to severe forms of calcification. Preoperative use of CT provides practical surgical information in patients with porcelain aorta who may otherwise experience catastrophic complications.

\section{Conflict of Interest}

The authors have no conflict of interest relevant to this publication.

Comment on this Article or Ask a Question 


\section{References}

1. van der Linden J, Hadjinikolaou L, Bergman P, Lindblom D. Postoperative stroke in cardiac surgery is related to the location and extent of atherosclerotic disease in the ascending aorta. J Am Coll Cardiol. 2001;38:131-135. DOI: 10.1016/S07351097(01)01328-6

2. Djaiani G, Fedorko L, Borger M, Mikulis D, Carroll J, Cheng D, et al. Mild to moderate atheromatous disease of the thoracic aorta and new ischemic brain lesions after conventional coronary bypass graft surgery. Stroke. 2004;35:e356-e358. DOI: 10.1161/01.STR.0000138783.63858.62

3. Tunich PA, Kronzon I. Atheromas of the thoracic aorta: clinical and therapeutic update. J Am Coll Cardiol. 2000;35:545-554. DOI: 10.1016/S0735-1097(99)00604-X

4. The French Study of Aortic Plaques in Stroke Group, Amarenco P, Cohen A, Hommel M, Moulin T, Leys $D$, et al. Atherosclerotic disease of the aortic arch as a risk factor for recurrent ischemic stroke. N Engl J Med. 1996;334:1216-2170. DOI: 10.1056/ NEJM199605093341902

5. Keeley EC, Grines CL. Scraping of aortic debris by coronary guiding catheters: a prospective evaluation of 1000 cases. J Am Coll Cardiol. 1998;32:1861-1865. DOI: 10.1016/S0735-1097(98)00497-5

6. Iribarren C, Sidney S, Sternfeld B, Browner WS. Calcification of the aortic arch: risk factors and association with coronary heart disease, stroke and peripheral vascular disease. JAMA. 2000;283:2810-2815. DOI: 10.1001/jama.283.21.2810

7. Eisen $A$, Tenenbaum A, Koren-Morag N, Tanne D, Shemesh J, Imazio M, et al. Calcification of the thoracic aorta as detected by spiral computed tomography among stable angina pectoris patients. Circulation. 2008;118:1328-1334. DOI: 10.1161/CIRCULATIONAHA.107.712141

8. Zingone B, Rauber E, Gatti G, Pappalardo $A$, Benussi B, Forti $G$, et al. Diagnosis and management of severe atherosclerosis of the ascending aorta. Eur J Cardiothorac Surg. 2007;31:990-997. DOI: 10.1016/j. ejcts.2007.02.017

9. Nishi $H$, Mitsuno $M$, Tanaka $H$, Ryomoto $M$, Fukui S, Miyamoto $\mathrm{Y}$. Who needs preoperative routine chest computed tomography for prevention of stroke in cardiac surgery? Interact Cardiovasc Thorac Surg. 2010;11:3033. DOI: $10.1510 /$ icvts.2009.231761

10. Selim M. Perioperative stroke. N Engl J Med. 2007;356:706-713. DOI: 10.1056/NEJMra062668

11. Palmerini T, Biondi-Zoccai G, Riva DD, Mariani A, Savini C, DiEusanio M, et al. Risk of stroke with percutaneous coronary intervention compared with on-pump and off-pump coronary artery bypass graft surgery: evidence from a comprehensive network meta-analysis. Am Heart J. 2013;165:910-917.e14. DOI: 10.1016/j.ahj.2013.03.011

12. Gulati R, Rihal CS, Gersh BJ. The SYNTAX trial: a perspective. Circ Cardiovasc Interv.
2009;2:463-467. DOI: 10.1161/CIRCINTERVENTIONS.109.882670

13. Lee E, Choi K, Ryu J, Jeon S, Lee S, Park S, et al. Stroke risk after coronary artery bypass graft surgery and extent of cerebral artery atherosclerosis. J Am Coll Cardiol. 2011;57:1811-1818. DOI: 10.1016/j. jacc.2010.12.026

14. Evangelista A, Flachskampf FA, Erbel R, Antonini-Canterin F, Vlachopoulos C, Rocchi G. Echocardiography in aortic diseases: EAE recommendations for clinical practice. Eur J Echocardiogr. 2010;11:645-658. DOI: 10.1093/ejechocard/jeq056

15. Amorim PA, Penov K, Lehmkuhl L, Haensig M, Mohr FW, Rastan AJ. Not all porcelain is the same: classification of circular aortic calcification (porcelain aorta) according to the impact on therapeutic approach. Thorac Cardiovasc Surg. 2013;61:559-563. DOI: 10.1055/s0032-1333204

Cite this article as: Diken $\mathrm{Al}$, Yalçınkaya A, Özyalçın S. Distribution of Thoracic Aortic Calcifications in Patients Undergoing Coronary Artery Bypass Grafting. AORTA (Stamford). 2017;5(5):132-138. DOI: https://doi. org/10.12945/j.aorta.2017.17.035

\section{EDITOR'S QUESTIONS}

\section{How did your findings alter your own strategy and practice for CABG? How do you recommend that it alter the practices of your readers?}

We observed very low incidence for postoperative stroke $(0.46 \%)$. The presence of preoperative data regarding the ascending aorta influenced preoperative decision-making in terms of site of arterial cannulation, potentially changing the operative approach, or perhaps influencing the decision for staged $\mathrm{PCl}$ in the setting of significantly unfavorable calcification. The use of CT may keep you and your patients on the safer side. 